\title{
Angiogenesis and lymphangiogenesis are downregulated in primary breast cancer
}

\section{E-M Boneberg', DF Legler', MM Hoefer', C Öhlschlegel ${ }^{2}$, H Steininger ${ }^{3}$, L Füzesi ${ }^{4}$, GM Beer ${ }^{5}$, V Dupont-Lampert ${ }^{6}$, F Otto $^{6}$, H-J Senn ${ }^{6}$ and G Fürstenberger ${ }^{*, 6}$}

'Biotechnology Institute Thurgau at the University of Konstanz, Kreuzlingen, Switzerland; ²Department of Pathology, Cantonal Hospital St Gallen, St Gallen, Switzerland; ${ }^{3}$ Department of Pathology, Municipal Hospital of Friedrichshafen, Friedrichshafen, Germany; ${ }^{4}$ Department of Pathology, Georg August University, Göttingen, Germany; ${ }^{5}$ Division for Plastic and Aesthetic Surgery, Bodenseeklinik Lindau, Lindau, Germany; ${ }^{6}$ Tumor and Breast Center ZeTuP, St Gallen, Switzerland

BACKGROUND: Angiogenesis and lymphangiogenesis are considered to play key roles in tumour growth, progression and metastasis. However, targeting tumour angiogenesis in clinical trials showed only modest efficacy. We therefore scrutinised the concept of tumour angiogenesis and lymphangiogenesis by analysing the expression of crucial markers involved in these processes in primary breast cancer.

METHODS: We analysed the expression of angiogenic, lymphangiogenic or antiangiogenic factors, their respective receptors and specific markers for endothelial and lymphendothelial cells by quantitative real-time RT-PCR in primary breast cancer and compared the expression profiles to non-cancerous, tumour-adjacent tissues and breast tissues from healthy women.

RESULTS: We found decreased mRNA amounts of major angiogenic and lymphangiogenic factors in tumour compared to healthy tissues, whereas antiangiogenic factors were upregulated. Concomitantly, angiogenic and lymphangiogenic receptors were downregulated in breast tumours. This antiangiogenic, antilymphangiogenic microenvironment was even more pronounced in aggressive tumours and accompanied by reduced amounts of endothelial and lymphatic endothelial cell markers.

CONCLUSION: Primary breast tumours are not a site of highly active angiogenesis and lymphangiogenesis. Selection for tumour cells that survive with minimal vascular supply may account for this observation in clinical apparent tumours.

British Journal of Cancer (2009) I I , 605-6I4. doi:I0.1038/sj.bjc.66052I9 www.bjcancer.com

(c) 2009 Cancer Research UK

Keywords: angiogenesis; lymphangiogenesis; real-time PCR; tumour microenvironment; breast cancer

Angiogenesis, the formation of new blood vessels from existing vasculature, is fundamental in tumour growth, progression and metastasis (Folkman, 1992). The regulation of tumour angiogenesis depends on a delicate balance of angiogenic and antiangiogenic factors, that can be secreted by both tumour and stroma cells. In the past years many different proteins have been identified as angiogenic activators. Foremost among them are members of the vascular endothelial growth factor (VEGF) family with seven members (VEGF-A, -B, -C, -D, -E, svVEGF and placenta growth factor; Takahashi and Shibuya, 2005). Further fibroblast growth factor-2 (FGF-2, FGF basic) and hepatocyte growth factor (HGF) have angiogenic activities (Bussolino et al, 1992; Folkman and Shing, 1992). In the angiopoietin family angiopoietin-1 (ANG1) and -2 (ANG-2) can influence the angiogenic process. They bind both to the receptor TIE-2, but their function in shaping angiogenesis is very complex and controversial: TIE- 2 signalling could promote or inhibit angiogenesis by influencing survival of endothelial cells, vessel growth and/or vessel maturation

*Correspondence: Dr G Fürstenberger, Tumor and Breast Center ZeTuP, Rorschacherstrasse 150, St Gallen 9006, Switzerland;

E-mail: guerstenberger@gr.zetup.ch

Received I April 2009; revised 3 July 2009; accepted 3 July 2009 depending on the balance of ANG-1 and ANG-2, the formation of different splice variants of the angiopoietins, the presence of soluble TIE receptors, formation of multimers of the angiopoietins or dimers of the receptors, interactions with integrins and the presence of other angiogenic factors such as VEGF-A in the microenvironment (reviewed in Shim et al, 2007).

Also, members of the platelet-derived growth factor (PDGF) family can promote angiogenesis as disulphide-linked heterodimers (PDGF-AB) or homodimers (PDGF-AA, PDGF-BB, PDGF-CC and PDGF-DD) (Yu et al, 2003). Moreover, the chemokines CXCL1, -2, -3, -5, -6, -7 and -8 can exert angiogenic activity (Strieter et al, 2006).

Angiogenesis, on the other hand, is also regulated by antiangiogenic factors. Two important angiostatic factors are angiostatin and endostatin, which are both produced by proteolytic cleavage of plasminogen or collagen XVIII, respectively (O'Reilly et al, 1994, 1997). Moreover, thrombospondin-1 (TSP-1) and thrombospondin-2 (TSP-2) and the chemokines CXCL4, CXCL9, CXCL10 and CXCL11 can inhibit angiogenesis (Lawler, 2000; Lasagni et al, 2003; Strieter et al, 2006).

Overexpression of angiogenic factors in human cancers has been described previously, for example, for VEGF-A in lung, breast and pancreas carcinoma (Yoshiji et al, 1996; Itakura et al, 2000; Merrick et al, 2005), for FGF-2 in pancreas carcinoma and prostate 
cancer (Yamanaka et al, 1993; Giri et al, 1999) and for ANG-1 and ANG-2 in gastric and hepatocellular carcinoma (Torimura et al, 2004; Wang et al, 2005). However, there are also reports that disagree with the concept that tumours produce higher amounts of angiogenic factors than their normal tissue counterparts: in breast cancer tissues the expression of FGF-2 was reduced (Luqmani et al, 1992) or did not differ from the expression in normal breast tissues (Colomer et al, 1997). Furthermore, VEGF-A was similarly expressed in breast cancer and normal tissues (Soufla et al, 2006). These inconsistent findings demonstrate that a general upregulation of angiogenic factors in tumours should not be regarded as a paradigm.

Besides their ability to induce angiogenesis, tumours can also induce the formation of new lymphatic vessels, a process referred to as lymphangiogenesis (Wissmann and Detmar, 2006; Karpanen and Alitalo, 2008). Lymphangiogenesis is mainly stimulated by VEGF-C and VEGF-D, and also VEGF-A, HGF as well as members of the FGF, angiopoietin, PDGF and insulin-like growth factor families can support this process.

Targeting tumour angiogenesis and tumour lymphangiogenesis are promising therapeutic strategies for the treatment of cancer and the inhibition of tumour cell dissemination. Antiangiogenic therapies have been transferred from preclinical to clinical application in recent years (Ellis and Hicklin, 2008; Kerbel, 2008), but their clinical efficacy is relatively modest and a therapeutic benefit of VEGF-targeted therapy probably involves multiple mechanisms, beside its antiangiogenic effect. Considering the conflicting data for the upregulation of angiogenic factors in tumours and the limited clinical success of antiangiogenic therapy, the paradigm of tumours as sites of highly active angiogenesis needs to be scrutinised. Especially, as most data analysing the expression of angiogenic factors in tumours are based on semiquantitative methods such as immunohistochemistry, RNAse protection assays, northern blots or conventional RT - PCR. Here, we re-evaluate the concept of tumour angiogenesis and tumour lymphangiogenesis with quantitative real-time RT-PCR for a comprehensive set of angiogenic, antiangiogenic and lymphangiogenic factors, their respective receptors and specific markers for endothelial cells or lymphatic endothelial cells in primary breast cancer tissues compared to expression profiles of non-cancerous, tumour-adjacent tissues and breast tissues from healthy women.

\section{PATIENTS AND METHODS}

\section{Patients and tissue samples}

The study was approved by the regional ethic board of the Canton of St Gallen, Switzerland (for breast cancer patients), the institutional ethic board of the University Hospital Göttingen, Germany (for clear cell renal carcinoma) and the Institutional Review Board of the University of Konstanz, Germany (for healthy controls) and all patients and controls gave written informed consent before study entry. We studied 48 female patients diagnosed with primary breast cancer and 12 healthy controls. The median ages were 62 years (range 39-89 years) for the patients and 37 years (range 18-65 years) for the controls. Patients did not receive any kind of cancer-related pretreatment before the surgical removal of the tumour. Breast cancer tissue was obtained from the middle ring of the tumour (see Supplementary Figure S1) in a macroscopically vital looking area of the tumour by the pathologist directly after surgical removal of the tumour. Additionally, tumour-adjacent tissue (distance to tumour $1-2 \mathrm{~cm}$ ) was collected from 41 of these patients. Samples from healthy women were obtained from breast tissue removed during plastic-aesthetic reduction surgery. Both types of normal breast tissue were macroscopically chosen to contain glandular breast tissue and not only fat tissue. To verify tumour tissue or tumour-free tissue, respectively, and the absence of necrotic areas cryosections were obtained from the selected tissue samples and histologically evaluated by the pathologist.

From the 48 analysed breast tumours, 6 tumours were assigned grade 1, 30 tumours grade 2 and 12 tumours grade 3 according to the Elston-modified Bloom and Richardson grading system; 33 tumours were classified as ductal, 8 as mixed ductal-lobular and 7 as lobular. Lymph node metastasis was detected in 21 patients and 41 of the breast cancers were classified as oestrogen receptorpositive. Overexpression of Her-2/neu was detected with FISH in six of the tumour tissues.

As a positive control for an angiogenic tumour type, tissues from clear cell renal carcinoma were analysed from five patients and compared to tumour-free tissue from the same kidney.

\section{RNA isolation and reverse transcription}

A small part of frozen breast tissue (approximately $3 \times 3 \times 3 \mathrm{~mm}$ ) was homogenised in QIAzol Lysis Reagent (Qiagen, Basel, Switzerland) and RNA was isolated with the RNeasy Kit plus additional Dnase digestion (Qiagen) according to the manufacturer's instructions. RNA was reverse transcribed with the High Capacity cDNA Archive Kit from Applied Biosystems (Rotkreuz, Switzerland).

\section{Real-time RT-PCR}

Real-time RT-PCR analysis was performed with custom designed TaqMan Low Density Arrays (Applied Biosystems) in 384-well micro fluidic cards. Details of the chosen assays are given in the Supplementary Table 1 online. PCR was performed with $100 \mathrm{ng}$ of total RNA converted to cDNA per port in Taqman Universal PCR Master Mix $(2 \times)$ (Applied Biosystems) in a 7900HT Fast real-time PCR System (Applied Biosystems) with a TaqMan Low Density Array Upgrade using the following thermal settings: 2 min at $50^{\circ} \mathrm{C}$, $10 \mathrm{~min}$ at $94.5^{\circ} \mathrm{C}$ and 40 cycles of $30 \mathrm{~s}$ at $97^{\circ} \mathrm{C}$, and $1 \mathrm{~min}$ at $59.7^{\circ} \mathrm{C}$. Data from real-time RT-PCR were normalised to the mean of four different housekeeping genes (B2M, GAPDH, UBC, TBP) and relative mRNA expression was calculated with the $\Delta \Delta C_{\mathrm{t}}$ method. As calibrator, a cDNA mixture of a tumour, a tumour-adjacent and a healthy control sample was used.

\section{Immunohistochemistry}

Deparaffinised and rehydrated sections $(4 \mu \mathrm{m})$ were microwaved, endogenous peroxidase was blocked and sections were incubated with an antibody to CD31 (clone JC/70A; 1:150 dilution; Dako, Hamburg, Germany) for $25 \mathrm{~min}$ at room temperature. Binding of the antibody was visualised with the Dako REAL Detection System, Peroxidase/DAB +, Rabbit/Mouse (Dako) and sections were counterstained with haematoxylin.

\section{ELISA measurement of growth factors in serum}

From 35 of the 48 breast cancer patients, serum had been collected at the time of diagnosis. Serum levels of growth factors were quantified by sandwich ELISA using the respective DuoSet ELISA Kits (R\&D Systems, Abingdon, United Kingdom) according to the manufacturer's instructions. Serum samples of 72 healthy women were used as a control group.

\section{Statistical analysis}

Statistical analysis was performed using GraphPad Instat (Instat Statistics, GraphPad Software). For comparison of tumour, tumour-adjacent and healthy tissues, two-sided unpaired nonparametric testing with the Kruskal-Wallis test and Dunn's multiple comparisons test as post test was performed. For the 

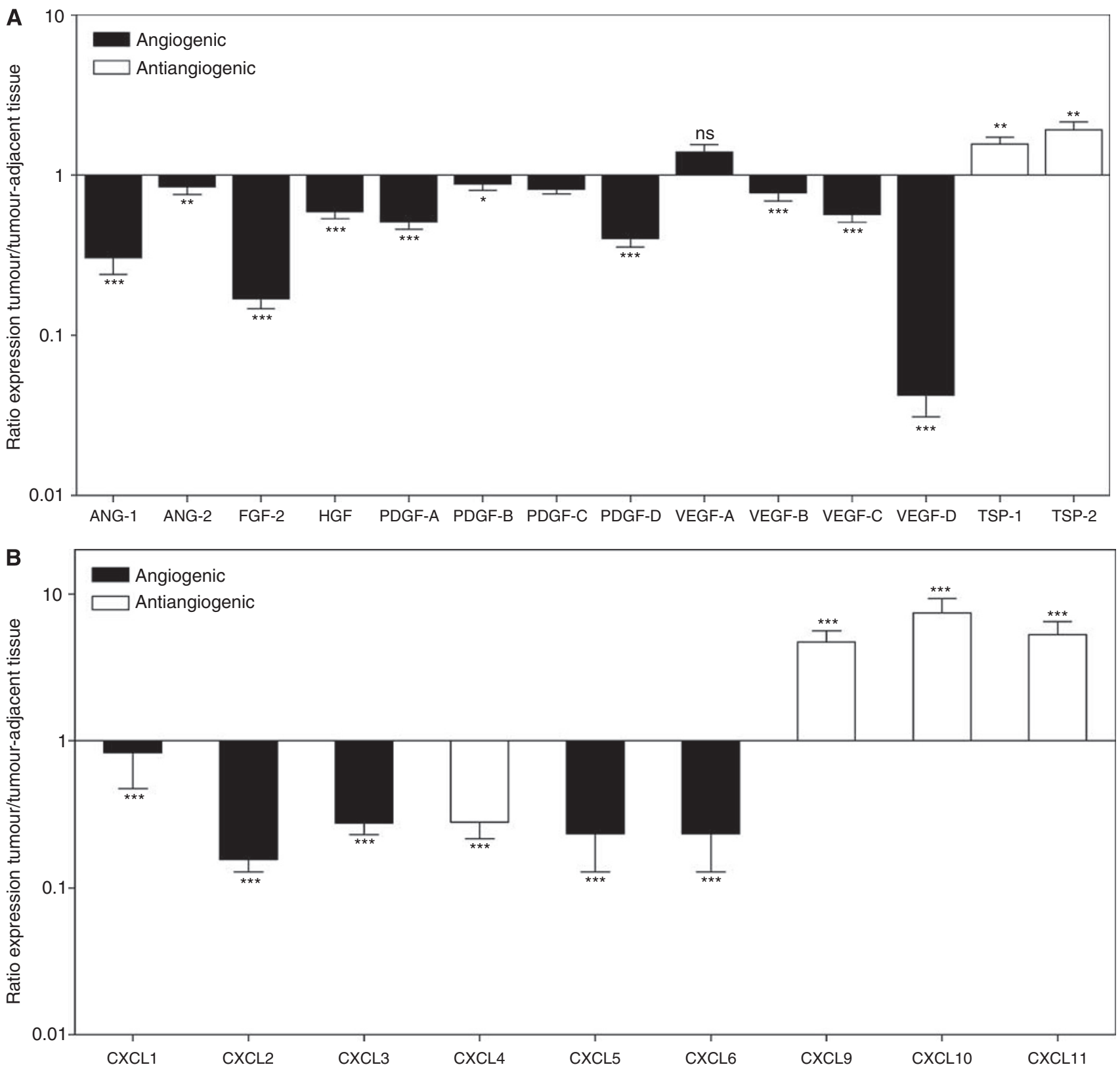

Figure I Expression of angiogenic, antiangiogenic and lymphangiogenic growth factors and chemokines in primary breast cancer. The mRNA expression of angiogenic, antiangiogenic and lymphangiogenic growth factors $(\mathbf{A})$ and chemokines $(\mathbf{B})$ was quantified by real-time RT-PCR in breast cancer tissues and tumour-adjacent tissues of the same patients. The ratio of the expression in tumour tissue to the respective tumour-adjacent tissue was calculated for each patient. The bars show the mean ratio with s.e.m. for 4 I patients. ${ }^{*} P<0.05$, ${ }^{*} * P<0.0$ I and ${ }^{*} * * P<0.00$ I.

comparison of tumour samples and tumour-adjacent tissues from the same patients paired non-parametric testing with the Wilcoxon signed rank test was performed. Differences were considered statistically significant at $P<0.05$.

\section{RESULTS}

Angiogenic and lymphangiogenic factors are downregulated in breast cancer tissues, whereas antiangiogenic factors are upregulated

To obtain a comprehensive profile of the major factors regulating angiogenesis and lymphangiogenesis in breast cancer we analysed their expression by quantitative real-time RT-PCR in 48 breast cancer tissues, 41 tumour-adjacent tissues and 12 breast tissues from healthy women. As the expression of housekeeping genes could vary considerably between tumour and healthy tissues and between different donors, we normalised the expression of all genes to the mean expression of four different housekeeping genes (GAPDH, UBC, TBP and B2M) to correct for these possible variations.

To compare the expression profiles of breast cancer and normal breast tissues, we analysed paired samples of normal and tumour tissues from 41 patients and calculated the ratio of the expression in a breast cancer sample to the expression in the tumour-adjacent tissue of the same patient. Compared to their respective tumouradjacent tissues, the breast cancer samples showed a significantly decreased mRNA expression of all measured angiogenic factors except for VEGF-A (Figure 1A). The mean expression of ANG-1 in 
tumour tissues was reduced to $30 \%$ of the expression levels found in the tumour-adjacent tissues, the expression of ANG-2 was decreased to $84 \%$, FGF- 2 to $17 \%$ and HGF to $59 \%$. The PDGF family was also downregulated in breast cancer samples: compared to tumour-adjacent tissues the mean expression of PDGF-A was $51 \%$, PDGF-B $87 \%$, PDGF-C $81 \%$ and PDGF-D $40 \%$ of the expression levels found in the tumour-adjacent tissues. VEGF-A was upregulated in 22 tumours (maximum 4.7-fold increase), in 15 tumours downregulated (minimum 3.9-fold decrease) and in 4 tumours expressed in equal amounts as in the tumour-adjacent tissues. There was no correlation between increased VEGF-A expression in tumours and lymph node metastasis, tumour grading or tumour size.

The expression of the other VEGF family members was downregulated in breast cancer tissues: VEGF-B was reduced to $77 \%$, VEGF-C to $56 \%$ and VEGF-D to $4 \%$ of the expression in the tumour-adjacent tissues.

The mRNA expression of angiogenic chemokines was also downregulated in breast cancer compared to tumour-adjacent tissues (Figure 1B): for CXCL1, a downregulation to $83 \%$ was observed in the tumour tissues; CXCL2 was reduced to $16 \%$; CXCL3 to $28 \%$; CXCL5 to $24 \%$ and CXCL6 to $24 \%$ of the expression levels in the tumour-adjacent tissues.

A significantly higher mRNA expression of all analysed antiangiogenic factors was found in the breast cancer samples compared to tumour-adjacent tissues or healthy tissues (Figure 1A and B), except for CXCL4, which was decreased to $28 \%$ in the tumour samples. For TSP-1 a 1.6-fold and for TSP-2 a 1.9-fold higher expression was found in the tumour tissues compared to tumour-adjacent samples. For the angiostatic chemokines, CXCL9, CXCL10 and CXCL11, an increased mean expression of 4.7-, 7.4and 5.3-fold, respectively, was observed in breast cancer samples compared to tumour-adjacent tissues.

Supplementary Figure S2 shows the individual expression of the measured factors and chemokines in all analysed samples. For these factors and chemokines, no significant difference between the median expression in tumour-adjacent tissues and breast tissues from healthy women could be found, except for VEGF-C, which showed a significant higher expression in tumour-adjacent tissues.

These data show that the mRNA expression of all angiogenic factors is downregulated in breast carcinoma with the exception of VEGF, which was expressed in comparable amounts in tumour tissues and tumour-adjacent tissues. Conversely, antiangiogenic factors were upregulated in the tumour. In conclusion, the mRNA expression profile of angiogenesis-regulating factors is shifted in the breast cancer microenvironment towards antiangiogenesis, and the major lymphangiogenic factors VEGF-C and VEGF-D are downregulated.

The observed antiangiogenic expression pattern in breast cancer tissues may be explained by two different hypotheses. Tumour progression may select for tumour cells able to grow without optimal vascular supply so that at the time of diagnosis and surgery the angiogenic microenvironment in the tumour could have disappeared. As an alternative model, the tumour microenvironment could become increasingly angiogenic as tumour progression proceeds and tumours become more aggressive. In this case the analysed tumours would have been excised before they had developed an angiogenic environment. To test these hypotheses we compared the median expression of more aggressive tumours with a histopathologic grading of 3 to less aggressive tumours with grade 1 (Figure 2). For FGF-2, we found significant lower mRNA levels (2.3-fold) in grade 3 tumours than in grade 1 tumours. For most other angiogenic factors, we observed a lower expression in grade 3 tumours than in grade 1 tumours but these differences were not significant. For VEGF-A, PDGF-D and the angiogenic chemokines, no difference was found between tumours with different grading. Interestingly, the antiangiogenic chemokines CXCL9, CXCL10 and CXCL11 were more expressed in grade 3 tumours than in grade 1 tumours. These data indicate that the antiangiogenic environment is more pronounced in aggressive tumours supporting the hypothesis that the observed antiangiogenic shift results from selection of tumour cells that can grow with low vascularisation and do not produce angiogenic factors anymore.

To verify the validity of our real-time RT-PCR approach we analysed clear cell renal carcinoma, a tumour type known to be highly angiogenic (Turner et al, 2002). As expected, we found a strong upregulation of VEGF-A in the tumour tissues (Supplementary Figure S3). Compared to normal kidney tissues, the median expression of VEGF-A was 8.9 -fold increased in the renal cancer tissues. Also, PDGF-D was significantly upregulated (3.7-fold) in renal cell carcinoma. ANG-2 mRNA was found in higher amounts in the tumour tissues, but this difference was not significant. VEGF-B, HGF and PDGF-C were downregulated in renal carcinoma, whereas the other analysed angiogenic and antiangiogenic factors were expressed in comparable amounts. This analysis showed that our real-time RT-PCR assay can also detect an angiogenic tumour microenvironment. Thus the observed antiangiogenic profile of breast cancer tissues cannot result from a technical shortcoming of the detection method.

The gene expression profiles showed a diminished expression of the main angiogenic and lymphangiogenic factors in breast cancer. To investigate whether this difference is also detectable on protein levels we performed ELISA measurements of the most important factors in homogenates of breast cancer tissues and normal tumour-adjacent tissues of the same donors (Supplementary Figure S4). Similar amounts of VEGF-A, FGF-2 and PDGF-AA were present in tumour and tumour-adjacent tissues. ANG-1 and VEGF-D were detected significantly less in breast cancer than in the tumour-adjacent tissues (3.2-fold and 2-fold less). The downregulation of FGF-2 and PDGF-A in the tumour tissues on mRNA level was not reflected in the protein data. As these cytokines are secreted soluble proteins, diffusion and transport by lymph or blood into the tumour tissue could balance the differences in protein production. The downregulation of ANG-1 and VEGF-D was also seen on protein level, but was not as pronounced as on the mRNA level.

\section{Receptors of angiogenesis and lymphangiogenesis are downregulated in breast cancer tissues}

For the regulation of angiogenesis, not only the expression of growth factors is important, but also the expression of the respective receptors. Therefore, the mRNA expression of receptors for angiogenic and lymphangiogenic factors was analysed by quantitative real-time RT-PCR and the ratio of the expression in a breast cancer sample to the expression in the tumour-adjacent tissue of the same patient was calculated (Figure 3). The VEGF receptors were downregulated in breast cancer tissues compared to the respective tumour-adjacent tissues: the mean VEGF-R1 (FLT1) expression in the tumour tissue was reduced to $47 \%$, VEGF-R2 (KDR) to $49 \%$ and VEGF-R3 (LT4) to $49 \%$ of the expression levels in tumour-adjacent tissues. For neuropilin-1 (NRP-1) and neuropilin-2 (NRP-2), which can act as enhancers for VEGF-R signalling, we found a mean decrease of NRP-1 in breast tumour tissue to $55 \%$ of the expression levels in tumour-adjacent tissues, whereas NRP-2 expression showed no significant difference between tumour and tumour-adjacent tissues. The angiopoietin receptor TIE-2 was reduced to $40 \%$ in breast cancer tissues compared to tumour-adjacent tissues. For the two PDGF receptors, PDGF-R $\alpha$ and PDGF-R $\beta$, the mean expression in breast cancer samples was reduced to 41 and $72 \%$ of the expression levels found in tumour-adjacent tissues. CXCR2, the receptor for angiogenic chemokines, was reduced in the breast cancer tissues to $56 \%$ of the 

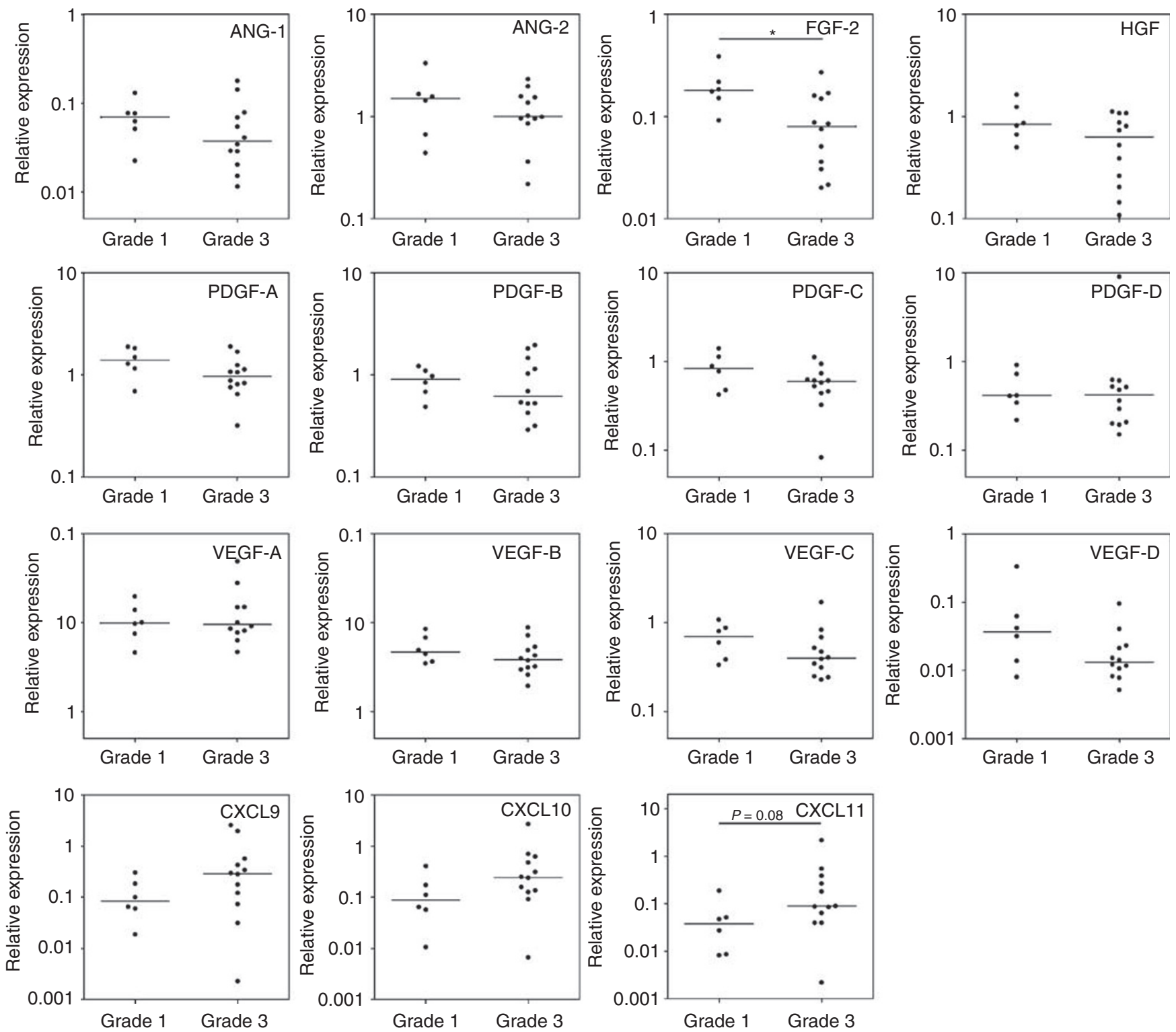

Figure 2 Comparison of expression levels of angiogenic, antiangiogenic and lymphangiogenic growth factors, and chemokines in primary breast cancer according to histopathologic grading. Relative expression data for the different growth factors and chemokines were analysed according to the histopathologic grading of the primary breast tumours. The horizontal line indicates the median of each group. $* P<0.05$.

level in the-adjacent tissues, whereas CXCR3, the receptor for angiostatic chemokines, was upregulated 3.4-fold.

Supplementary Figure S5 shows the individual expression of the measured receptors in all analysed samples. For these receptors no significant difference between the median expression in tumouradjacent tissues and breast tissues from healthy women could be found.

Concomitant with the reduced expression of angiogenic factors, the expression of angiogenesis-inducing receptors also was downregulated in the breast tumours. Moreover, the major lymphangiogenic receptor VEGF-R3 was downregulated.

\section{Markers for endothelial and lymphatic endothelial cells are downregulated in breast cancer tissues}

It has been previously described that the measurement of the expression of endothelial markers such as CD146 by quantitative real-time PCR in tumour samples correlated with microvessel density assessed by immunohistology (Loges et al, 2007). We therefore determined the amount of endothelial cells in tumours or normal tissues by real-time RT-PCR analysis of two endothelial markers, CD146 and VE-Cadherin and lymphatic endothelial cells by analysing the expression of two specific lymphatic endothelial markers, lymphatic vessel endothelial hyaluronan receptor 1 (LYVE1) and Prospero-related homeobox 1 (PROX1; Figure 4A). The endothelial cell markers CD146 (MCAM) and VE-Cadherin showed a decreased expression in the tumour tissues compared to tumour-adjacent or healthy breast tissues: for CD146 the mean expression in tumour tissues was reduced to $44 \%$, and for VE-Cadherin to $40 \%$ of the expression levels in the respective tumour-adjacent tissues. The distribution of blood vessels in tumour and tumour-adjacent breast tissues was also analysed by immunohistochemical staining for CD31 in paraffin sections (Figure $4 \mathrm{~B}$ ). The CD31 staining confirmed the real-time RT-PCR data: also, the 


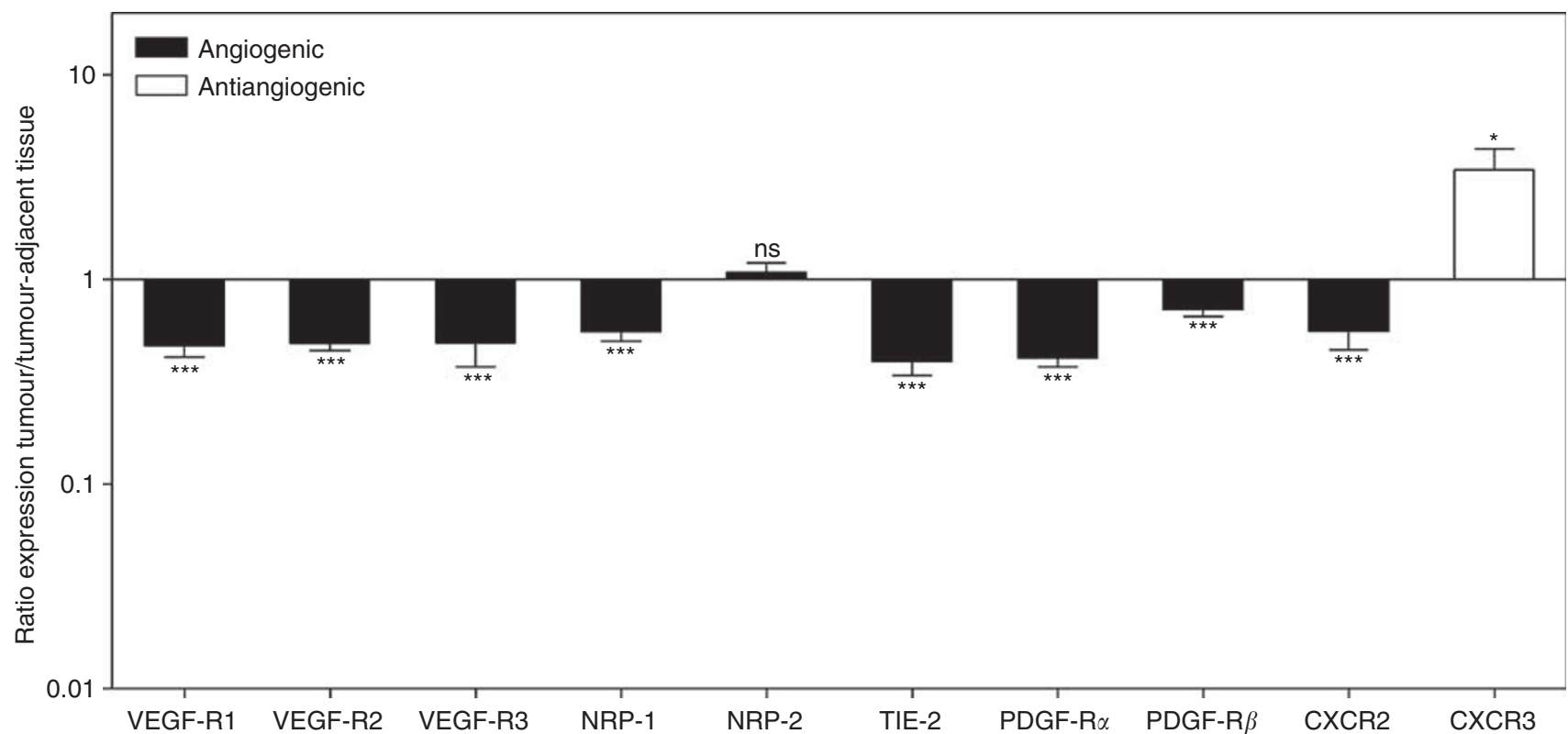

Figure 3 Expression of receptors for angiogenic and lymphangiogenic factors in primary breast cancer. The mRNA expression of angiogenic and lymphangiogenic receptors was quantified by real-time RT-PCR in breast cancer tissues and tumour-adjacent tissues of the same patients. The ratio of the expression in tumour tissue to the respective tumour-adjacent tissue was calculated for each patient. The bars show the mean ratio with s.e.m. for $4 \mathrm{I}$ patients. $* P<0.05$ and $* * * P<0.001$.

immunohistochemical detection of blood vessels showed a decreased vessel density in the tumour compared to the normal breast tissues.

Also, both lymphatic endothelial markers, LYVE1 and PROX1, were less expressed in the tumour compared to tumour-adjacent tissues (Figure 4A): for PROX1, the mean expression in the tumours was reduced to $21 \%$, for LYVE1 even to $6 \%$ of the expression levels in the tumour-adjacent tissues. These data indicate that the amounts of endothelial and lymphatic endothelial cells in breast tumour tissues are lower than those in tumouradjacent or healthy breast tissues and reflect the reduced amounts of angiogenic and lymphangiogenic factors and the increased expression of antiangiogenic factors in the breast cancer samples.

Supplementary Figure S6 shows the individual expression of the measured markers in all analysed samples. For these markers no significant difference between the median expression in tumouradjacent tissues and breast tissues from healthy women could be found.

Noteworthy, grade 3 tumours showed a lower expression of CD146, VE-Cadherin, LYVE1 and PROX1 than grade 1 tumours indicating that the amount of endothelial cells and lymph endothelial cells is further reduced with increasing tumour aggressiveness (Supplementary Figure S7).

The suitability of the selected endothelial markers in the realtime RT-PCR assay to assess tissue vascularisation was confirmed with the clear cell renal carcinoma tissues (Supplementary Figure S8). These highly vascularised tumours (Turner et al, 2002) showed a significant increase in the amounts of CD146 (fourfold) and VE-Cadherin (twofold) mRNA in comparison to normal kidney tissues reflecting the higher numbers of endothelial cells in the tumours.

\section{Angiogenic factors are decreased in the sera of breast cancer patients whereas antiangiogenic factors are elevated}

The levels of angiogenic factors in blood have been considered as surrogate markers for tumour angiogenesis. We therefore analysed the concentration of several angiogenesis-regulating factors in the sera of 35 breast cancer patients at the time of diagnosis and compared them to serum levels found in 72 healthy women.
ANG-1, HGF and PDGF-AB were found in significant lower levels in the sera of breast cancer patients than in healthy controls (Figure 5). VEGF-A was present in similar amounts in the sera of patients and controls. However, endostatin, a potent angiostatic factor, was significantly increased (1.4-fold) in the sera of breast cancer patients. This increase has been described before (Kuroi et al, 2001) and further corroborates the finding that the production of antiangiogenic factors is elevated in breast tumours. The concentrations of ANG-2 and FGF-2 were below the detection limits of the respective ELISAs. Our real-time RT-PCR data demonstrate that breast tumours do not produce increased amounts of angiogenic factors. In accordance to these data, we found no elevated levels of these factors in the sera of patients. Taken together, these findings indicate that primary breast tumours are not a significant source of angiogenic growth factors. In contrast, the tumours release angiostatic factors as endostatin. These tumour-derived angiostatic molecules could be responsible for the systemic downregulation of angiogenic factors as ANG-1, HGF or PDGF-AB, which were decreased in the sera of breast cancer patients.

\section{DISCUSSION}

Angiogenesis is considered as a limiting factor in tumour growth. Cancer cells require access to blood vessels for adequate supply of oxygen and nutrients. To gain this access, tumour and stroma cells can release mediators in the neoplastic microenvironment to induce migration and proliferation of endothelial cells resulting in the formation of new blood vessels. We studied the expression of these mediators and their respective receptors in primary breast cancer by quantitative real-time RT-PCR. Our data showed reduced expression of most angiogenesis-inducing factors in breast cancer tissues compared to non-cancerous, tumouradjacent tissues and breast tissues from healthy women, whereas angiogenesis inhibitors were expressed in higher amounts in the tumours. VEGF-A was the only angiogenic factor expressed in similar amounts in tumour tissues and normal tissues. These 
A
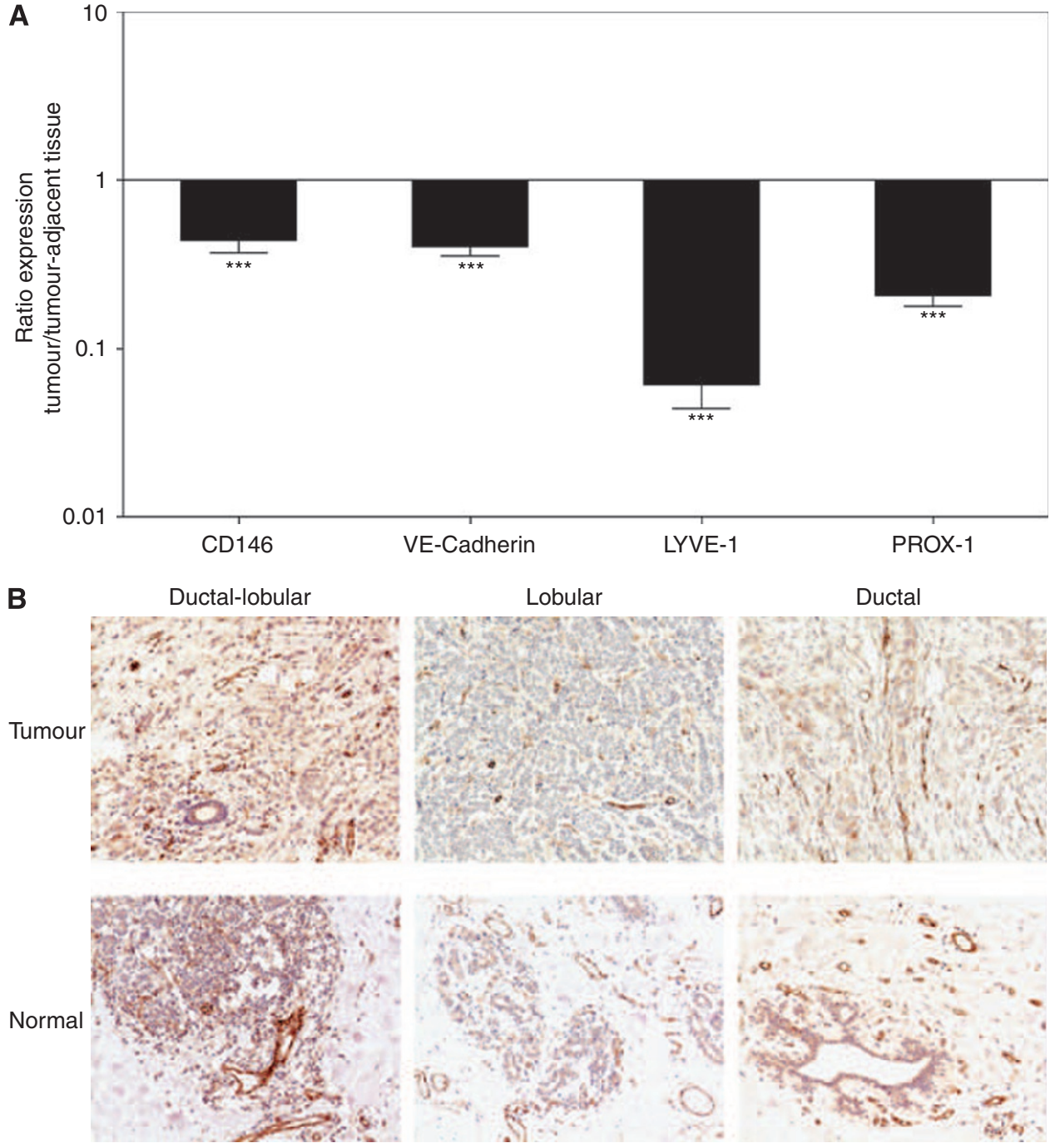

Figure 4 Endothelial and lymphatic endothelial cells in primary breast cancer. (A) The mRNA expression of markers for endothelial cells (CDI46, VE-Cadherin) and lymphatic endothelial cells (LYVEI, PROXI) was quantified by real-time RT-PCR in breast cancer tissues and tumour-adjacent tissues of the same patients. The ratio of the expression in tumour tissue to the respective tumour-adjacent tissue was calculated for each patient. The bars show the mean ratio with s.e.m. for $4 \mathrm{I}$ patients. $* * * P<0.00 \mathrm{I}$. (B) Immunohistochemical detection of endothelial cells in different breast cancer samples and corresponding normal breast tissue. Tissue samples were stained for CD3I with DAB (brown) and counterstained with haematoxylin (blue). Magnification $\times 10$.
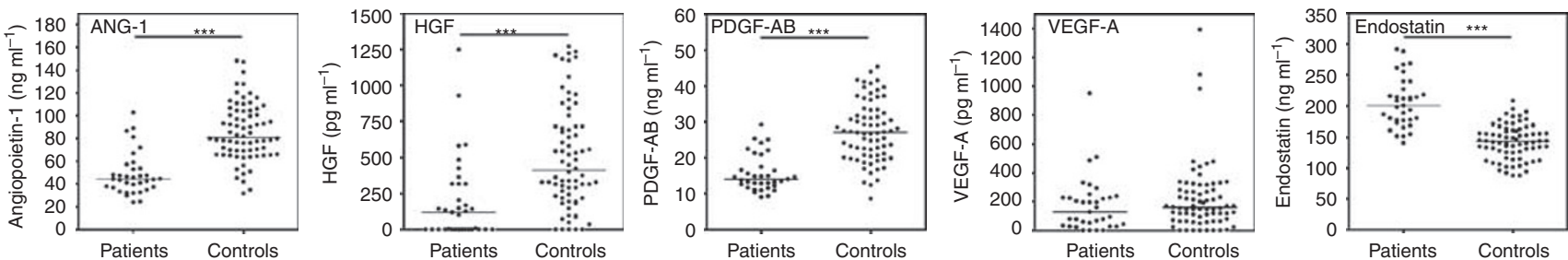

Figure 5 Quantification of angiogenic and antiangiogenic growth factors in serum. The concentration of angiogenic and antiangiogenic factors were determined by ELISA in the sera of breast cancer patients at the time of diagnosis and the sera of healthy controls. The horizontal line indicates the median of each group. ${ }^{*} * * P<0.001$.

findings were unexpected because tumours are generally considered as sites of active angiogenesis with high expression of angiogenic factors. Especially, high expression of VEGF-A is expected in tumours because the transcription of VEGF-A is increased by the transcription factor hypoxia-inducible factor 1 (HIF-1), which is stabilised in the hypoxic tumour environment (Shweiki et al, 1992; Liu et al, 1995).
Our real-time RT-PCR analysis revealed that not only the balance of angiogenic and antiangiogenic factors was shifted towards antiangiogenesis, but also the receptors for the angiogenic factors were expressed to a lesser extent in the cancer tissues than in normal tissues. All three VEGF receptors (VEGF-R1, -R2 and -R3) were downregulated as well as its coreceptor NRP-1, both PDGF receptors and the chemokine receptor CXCR2, which is 
activated by angiogenic chemokines. This receptor downregulation in the tumours is expected to result in a reduced responsiveness towards angiogenic stimuli. On the other hand, the chemokine receptor CXCR3, the receptor for angiostatic chemokines CXCL4, CXCL9, CXCL10 and CXCL11, was upregulated.

The antiangiogenic microenvironment and the downregulation of angiogenic receptors in the tumours were also reflected in the reduced number of endothelial cells. It may be counterintuitive that tumours exhibit lower microvessel densities than the corresponding normal tissues, but this has been described for breast, lung, renal cell and colon carcinomas (Eberhard et al, 2000). An immunohistochemical analysis showed a 35\% higher microvessel density in corresponding normal breast tissues compared to mammary carcinoma (Eberhard et al, 2000). This finding could be explained by the lower oxygen consumption rate of tumour cells (Steinberg et al, 1997) and their resistance to apoptosis under hypoxic conditions (Graeber et al, 1996). As tumour cells are viable at lower oxygen concentrations, they can survive at greater distances from the vasculature than normal cells (Hlatky et al, 2002). The observed antiangiogenic tumour environment and decreased number of endothelial cells in breast cancer may result from a constant selection process during disease progression for tumour cells tolerating minimal vascular supply. For these tumour cells, the expression of angiogenic factors is dispensable. Consequently, at the time of diagnosis and surgery the angiogenic microenvironment in the tumour could have disappeared. This model is supported by the fact that more aggressive tumours (grade 3) showed an even less angiogenic expression profile and less endothelial cells than grade 1 tumours. The strict dependence of tumour growth on neoangiogenesis could be limited to the early phase of tumour growth, during the 'angiogenic switch'. Once the tumour has established, tumour cells can grow into solid neoplasms by exploiting the host's preexisting vessels, without the need for new blood vessel formation, especially in vessel-dense organs. This 'co-opting' of vessels could lead to tumour progression even in the absence of neoangiogenesis (Leenders et al, 2004).

Data that show highly activated angiogenesis in tumours are mainly data from mouse tumour models and the upregulated angiogenesis in these tumours could be a specialty of these animal models. The subcutaneous space where experimental tumours are generally transplanted is essentially avascular, and tumours are therefore forced to induce angiogenesis to grow in this anatomical location (van Kempen and Leenders, 2006). Due to this experimental preselection of angiogenesis-competent tumours these mouse models may not reflect angiogenesis in natural growing tumours. It remains to be clarified if the antiangiogenic and antilymphangiogenic tumour microenvironment of primary human breast cancer is also found in tumours of other organs and in the metastases of the primary tumours.

Studies in animal tumour models and clinicopathological data have indicated that lymphangiogenesis in the vicinity of solid tumours may contribute to lymphatic metastasis (Skobe et al, 2001; Mattila et al, 2002; Nakamura et al, 2005). An activation of lymphangiogenesis by tumour-derived factors might be of high importance in breast cancer because it metastasises primarily via lymphatic vessels. Our data showed a reduced expression of the lymphangiogenic factors, VEGF-C and VEGF-D, and their respective receptor VEGF-R3 in the breast cancer tissues compared to normal breast tissues. In addition, other lymphangiogenic and angiogenic factors as FGF-2, HGF, angiopoietins and members of the PDGF family were downregulated. The diminished production of lymphangiogenic factors was also reflected by a reduced amount of lymphatic endothelial cells in the tumour samples. These data indicate that lymphangiogenesis is not increased in breast cancer tissues, but is even less activated than in normal breast tissues. This finding is in agreement with several other reports about lymphangiogenesis in human breast cancer: a reduction or even absence of lymphatic vessels has been described in the tumour area compared to normal breast tissues; also, a proliferative activity of the lymph vessels in the tumours was not detectable (Williams et al, 2003; Vleugel et al, 2004; Agarwal et al, 2005). For VEGF-C we found elevated expression in the tumour-adjacent tissues. But there was no correlation between VEGF-C expression in tumour-adjacent tissues and lymph node metastasis.

A previous study showed with immunohistochemistry an elevated number of VEGF-R3-positive blood vessels in breast tumours compared to corresponding normal breast tissues (Valtola et al, 1999). Further, the immunostaining for VEGF-C varied strongly among different invasive carcinoma samples, with some samples showing strong expression of VEGF-C whereas other stained only very weak or not at all. These findings can be reconciled with our data. With the real-time RT-PCR data we found a downregulation of VEGF-R3 expression in the total tumour mass, we cannot distinguish between the expression in the different cell types. As we see a reduction of endothelial cells in the tumour tissues, the decreased expression of VEGF-R3 (and those of other angiogenic receptors) could be due to the lower amounts of endothelial cells and not due to a downregulation per endothelial cell. Even an upregulation of VEGF-R3 per endothelial cell, but reduced numbers of endothelial cells in total, could lead to this result.

Our data show that the production of angiogenic and lymphangiogenic factors is reduced in human breast cancer tissues, and accordingly also the amounts of endothelial and lymphatic endothelial cells in the tumour. The antiangiogenic environment in breast tumours has several pivotal clinical implications. First, this finding could explain the modest efficacy of anti-VEGF therapies in breast cancer. Combination of standard chemotherapies with a VEGF-neutralising antibody (bevacizumab) increased response rates or prolonged progression-free survival, but did not prolong overall survival in metastatic breast cancer patients (Miller et al, 2005, 2007). As primary breast tumours seem to grow without an upregulated expression of VEGF-A, blockade of VEGF-A signalling cannot be expected to strongly impair further tumour growth. As anti-VEGF therapies can also induce 'normalisation' of tumour vessels and thus improve delivery of chemotherapeutics, this alternative mode of action could account for the observed clinical benefit in combination with standard chemotherapy (Jain, 2005; Dickson et al, 2007).

Further we have shown that primary breast tumours are predominantly a source of antiangiogenic factors and do not produce high amounts of angiogenic factors. An elimination of the primary tumour would reduce the amounts of antiangiogenic factors in the blood, whereas the formation of angiogenic factors would be stimulated after surgery in the wound healing process. The shift in the angiogenic balance could lead to a systemic activation of angiogenesis flaring up neovascularisation for micrometastases and thus breaking their dormant state. An activated outgrowth of metastases after the removal of the primary tumour has been described in animal tumour models and cancer patients (O'Reilly et al, 1994; Peeters et al, 2006). It was also reported that the surgical removal of primary colorectal cancers resulted in decreased plasma levels of angiostatin and endostatin and an increased metabolic activity of liver metastases indicating vessel neoformation (Peeters et al, 2005). To substitute the tumour-derived antiangiogenic factors, a long-term therapy with antiangiogenic factors could be a promising strategy to limit progression of the disease after surgery.

\section{ACKNOWLEDGEMENTS}

We thank Professor Robert A Weinberg (Whitehead Institute for Biomedical Research, Cambridge, MA) for critical reading of the article, conceptual advice and helpful experimental suggestions. The excellent technical assistance of Karin Ryffel, Elisabeth Bächler 
and Dora Spiess-Abbondandolo from the Tumor and Breast Center $\mathrm{ZeTuP}$ is greatly appreciated. This work was supported by grants of the Stiftung für Forschung in Tumordiagnostik und Prävention (STIFTUP), St Gallen, Switzerland, the Eugen \& Elisabeth Schellenberg Stiftung, Frauenfeld, Switzerland; the Thurgauische Stiftung für Wissenschaft und Forschung, and the Swiss State Secretariat for

\section{REFERENCES}

Agarwal B, Saxena R, Morimiya A, Mehrotra S, Badve S (2005) Lymphangiogenesis does not occur in breast cancer. Am J Surg Pathol 29: $1449-1455$

Bussolino F, Di Renzo MF, Ziche M, Bocchietto E, Olivero M, Naldini L, Gaudino G, Tamagnone L, Coffer A, Comoglio PM (1992) Hepatocyte growth factor is a potent angiogenic factor which stimulates endothelial cell motility and growth. J Cell Biol 119: 629-641

Colomer R, Aparicio J, Montero S, Guzmán C, Larrodera L, Cortés-Funes H (1997) Low levels of basic fibroblast growth factor (bFGF) are associated with a poor prognosis in human breast carcinoma. $\mathrm{Br} J$ Cancer 76: $1215-1220$

Dickson PV, Hamner JB, Sims TL, Fraga CH, Ng CY, Rajasekeran S, Hagedorn NL, McCarville MB, Stewart CF, Davidoff AM (2007) Bevacizumab-induced transient remodeling of the vasculature in neuroblastoma xenografts results in improved delivery and efficacy of systemically administered chemotherapy. Clin Cancer Res 13: 3942- 3950

Eberhard A, Kahlert S, Goede V, Hemmerlein B, Plate KH, Augustin HG (2000) Heterogeneity of angiogenesis and blood vessel maturation in human tumors: implications for antiangiogenic tumor therapies. Cancer Res 60: $1388-1393$

Ellis LM, Hicklin DJ (2008) VEGF-targeted therapy: mechanisms of antitumour activity. Nat Rev Cancer 8: 579-591

Folkman J (1992) The role of angiogenesis in tumor growth. Semin Cancer Biol 3: $65-71$

Folkman J, Shing Y (1992) Angiogenesis. J Biol Chem 267: 10931 - 10934

Giri D, Ropiquet F, Ittmann M (1999) Alterations in expression of basic fibroblast growth factor (FGF) 2 and its receptor FGFR-1 in human prostate cancer. Clin Cancer Res 5: $1063-1071$

Graeber TG, Osmanian C, Jacks T, Housman DE, Koch CJ, Lowe SW, Giaccia AJ (1996) Hypoxia-mediated selection of cells with diminished apoptotic potential in solid tumours. Nature 379: 88-91

Hlatky L, Hahnfeldt P, Folkman J (2002) Clinical application of antiangiogenic therapy: microvessel density, what it does and doesn't tell us. J Natl Cancer Inst 94: 883-893

Itakura J, Ishiwata T, Shen B, Kornmann M, Korc M (2000) Concomitant over-expression of vascular endothelial growth factor and its receptors in pancreatic cancer. Int J Cancer 85: 27-34

Jain RK (2005) Normalization of tumor vasculature: an emerging concept in antiangiogenic therapy. Science 307: 58-62

Karpanen T, Alitalo K (2008) Molecular biology and pathology of lymphangiogenesis. Annu Rev Pathol 3: 367-397

Kerbel RS (2008) Tumor angiogenesis. $N$ Engl J Med 358: 2039-2049

Kuroi K, Tanaka C, Toi M (2001) Circulating levels of endostatin in cancer patients. Oncol Rep 8: 405-409

Lasagni L, Francalanci M, Annunziato F, Lazzeri E, Giannini S, Cosmi L, Sagrinati C, Mazzinghi B, Orlando C, Maggi E, Marra F, Romagnani S, Serio M, Romagnani P (2003) An alternatively spliced variant of CXCR3 mediates the inhibition of endothelial cell growth induced by IP-10, Mig, and I-TAC, and acts as functional receptor for platelet factor 4. J Exp Med 197: $1537-1549$

Lawler J (2000) The functions of thrombospondin-1 and-2. Curr Opin Cell Biol 12: 634-640

Leenders WP, Küsters B, Verrijp K, Maass C, Wesseling P, Heerschap A, Ruiter D, Ryan A, de Waal R (2004) Antiangiogenic therapy of cerebral melanoma metastases results in sustained tumor progression via vessel co-option. Clin Cancer Res 10: 6222-6230

Liu Y, Cox SR, Morita T, Kourembanas S (1995) Hypoxia regulates vascular endothelial growth factor gene expression in endothelial cells. Identification of a $5^{\prime}$ enhancer. Circ Res 77: 638-643

Loges S, Clausen H, Reichelt U, Bubenheim M, Erbersdobler A, Schurr P, Yekebas E, Schuch G, Izbicki J, Pantel K, Bokemeyer C, Fiedler W (2007) Determination of microvessel density by quantitative real-time PCR in esophageal cancer: correlation with histologic methods, angiogenic
Education and Research. DFL is a recipient of a career development award from the Professor Max Cloëtta Foundation.

Supplementary Information accompanies the paper on British Journal of Cancer website (http://www.nature.com/bjc)

growth factor expression, and lymph node metastasis. Clin Cancer Res 13: $76-80$

Luqmani YA, Graham M, Coombes RC (1992) Expression of basic fibroblast growth factor, FGFR1 and FGFR2 in normal and malignant human breast, and comparison with other normal tissues. Br J Cancer 66: $273-280$

Mattila MM, Ruohola JK, Karpanen T, Jackson DG, Alitalo K, Härkönen PL (2002) VEGF-C induced lymphangiogenesis is associated with lymph node metastasis in orthotopic MCF-7 tumors. Int $J$ Cancer 98: 946-951

Merrick DT, Haney J, Petrunich S, Sugita M, Miller YE, Keith RL, Kennedy TC, Franklin WA (2005) Overexpression of vascular endothelial growth factor and its receptors in bronchial dypslasia demonstrated by quantitative RT-PCR analysis. Lung Cancer 48: 31 - 45

Miller K, Wang M, Gralow J, Dickler M, Cobleigh M, Perez EA, Shenkier T, Cella D, Davidson NE (2007) Paclitaxel plus bevacizumab vs paclitaxel alone for metastatic breast cancer. $N$ Engl $J$ Med 357: $2666-2676$

Miller KD, Chap LI, Holmes FA, Cobleigh MA, Marcom PK, Fehrenbacher L, Dickler M, Overmoyer BA, Reimann JD, Sing AP, Langmuir V, Rugo HS (2005) Randomized phase III trial of capecitabine compared with bevacizumab plus capecitabine in patients with previously treated metastatic breast cancer. J Clin Oncol 23: 792-799

Nakamura Y, Yasuoka H, Tsujimoto M, Imabun S, Nakahara M, Nakao K, Nakamura M, Mori I, Kakudo K (2005) Lymph vessel density correlates with nodal status, VEGF-C expression, and prognosis in breast cancer. Breast Cancer Res Treat 91: 125-132

O’Reilly MS, Boehm T, Shing Y, Fukai N, Vasios G, Lane WS, Flynn E, Birkhead JR, Olsen BR, Folkman J (1997) Endostatin: an endogenous inhibitor of angiogenesis and tumor growth. Cell 88: 277-285

O'Reilly MS, Holmgren L, Shing Y, Chen C, Rosenthal RA, Moses M, Lane WS, Cao Y, Sage EH, Folkman J (1994) Angiostatin: a novel angiogenesis inhibitor that mediates the suppression of metastases by a Lewis lung carcinoma. Cell 79: 315-328

Peeters CF, de Geus LF, Westphal JR, de Waal RM, Ruiter DJ, Wobbes T, Oyen WJ, Ruers TJ (2005) Decrease in circulating anti-angiogenic factors (angiostatin and endostatin) after surgical removal of primary colorectal carcinoma coincides with increased metabolic activity of liver metastases. Surgery 137: 246-249

Peeters CF, de Waal RM, Wobbes T, Westphal JR, Ruers TJ (2006) Outgrowth of human liver metastases after resection of the primary colorectal tumor: a shift in the balance between apoptosis and proliferation. Int J Cancer 119: 1249-1253

Shim WS, Ho IA, Wong PE (2007) Angiopoietin: a TIE(d) balance in tumor angiogenesis. Mol Cancer Res 5: 655-665

Shweiki D, Itin A, Soffer D, Keshet E (1992) Vascular endothelial growth factor induced by hypoxia may mediate hypoxia-initiated angiogenesis. Nature 359: $843-845$

Skobe M, Hawighorst T, Jackson DG, Prevo R, Janes L, Velasco P, Riccardi L, Alitalo K, Claffey K, Detmar M (2001) Induction of tumor lymphangiogenesis by VEGF-C promotes breast cancer metastasis. Nat Med 7: $192-198$

Soufla G, Porichis F, Sourvinos G, Vassilaros S, Spandidos DA (2006) Transcriptional deregulation of VEGF, FGF2, TGF-beta1, 2, 3 and cognate receptors in breast tumorigenesis. Cancer Lett 235: $100-113$

Steinberg F, Röhrborn HJ, Otto T, Scheufler KM, Streffer C (1997) NIR reflection measurements of hemoglobin and cytochrome aa3 in healthy tissue and tumors. Correlations to oxygen consumption: preclinical and clinical data. Adv Exp Med Biol 428: 69-77

Strieter RM, Burdick MD, Mestas J, Gomperts B, Keane MP, Belperio JA (2006) Cancer CXC chemokine networks and tumour angiogenesis. Eur J Cancer 42: $768-778$ 
Takahashi H, Shibuya M (2005) The vascular endothelial growth factor (VEGF)/VEGF receptor system and its role under physiological and pathological conditions. Clin Sci (Lond) 109: 227-241

Torimura T, Ueno T, Kin M, Harada R, Taniguchi E, Nakamura T, Sakata R, Hashimoto O, Sakamoto M, Kumashiro R, Sata M, Nakashima O, Yano H, Kojiro M (2004) Overexpression of angiopoietin-1 and angiopoietin-2 in hepatocellular carcinoma. J Hepatol 40: 799-807

Turner KJ, Moore JW, Jones A, Taylor CF, Cuthbert-Heavens D, Han C, Leek RD, Gatter KC, Maxwell PH, Ratcliffe PJ, Cranston D, Harris AL (2002) Expression of hypoxia-inducible factors in human renal cancer: relationship to angiogenesis and to the von Hippel-Lindau gene mutation. Cancer Res 62: 2957-2961

Valtola R, Salven P, Heikkilä P, Taipale J, Joensuu H, Rehn M, Pihlajaniemi T, Weich H, deWaal R, Alitalo K (1999) VEGFR-3 and its ligand VEGF-C are associated with angiogenesis in breast cancer. Am J Pathol 154: 1381 - 1390

van Kempen LC, Leenders WP (2006) Tumours can adapt to antiangiogenic therapy depending on the stromal context: lessons from endothelial cell biology. Eur J Cell Biol 85: 61-68

Vleugel MM, Bos R, van der Groep P, Greijer AE, Shvarts A, Stel HV, van der Wall E, van Diest PJ (2004) Lack of lymphangiogenesis during breast carcinogenesis. J Clin Pathol 57: 746 - 751

Wang J, Wu K, Zhang D, Tang H, Xie H, Hong L, Pan Y, Lan M, Hu S, Ning X, Fan D (2005) Expressions and clinical significances of angiopoietin-1, -2 and Tie2 in human gastric cancer. Biochem Biophys Res Commun 337: 386-393

Williams CS, Leek RD, Robson AM, Banerii S, Prevo R, Harris AL, Jackson DG (2003) Absence of lymphangiogenesis and intratumoural lymph vessels in human metastatic breast cancer. J Pathol 200: 195-206

Wissmann C, Detmar M (2006) Pathways targeting tumor lymphangiogenesis. Clin Cancer Res 12: 6865-6868

Yamanaka Y, Friess H, Buchler M, Beger HG, Uchida E, Onda M, Kobrin MS, Korc M (1993) Overexpression of acidic and basic fibroblast growth factors in human pancreatic cancer correlates with advanced tumor stage. Cancer Res 53: 5289-5296

Yoshiji H, Gomez DE, Shibuya M, Thorgeirsson UP (1996) Expression of vascular endothelial growth factor, its receptor, and other angiogenic factors in human breast cancer. Cancer Res 56: 2013-2016

Yu J, Ustach C, Kim HR (2003) Platelet-derived growth factor signaling and human cancer. J Biochem Mol Biol 36: 49-59

cC) $\$$ This work is licensed under the Creative Commons BY NC ND Attribution-NonCommercial-NoDerivs 3.0 License. To view a copy of this license, visit http://creativecommons.org/ licenses/by-nc-nd/3.0/. 